\title{
Carvedilol Matrix Tablet: Formulation and In Vitro Assessment
}

\author{
Joya Boidya $^{1}$, Ikramul Hasan ${ }^{2}$ and Md. Selim Reza ${ }^{2}$ \\ ${ }^{1}$ Department of Pharmacy, University of Asia Pacific, 74/A, Green Road, Dhaka-1205, Bangladesh \\ ${ }^{2}$ Department of Pharmaceutical Technology, Faculty of Pharmacy, University of Dhaka \\ Dhaka-1000, Bangladesh
}

(Received: December 30, 2019; Accepted: January 28, 2020; Published: January 30, 2020)

\begin{abstract}
The present study was conducted for preparing and assessing different in-vitro characteristics of hydrophilic polymer based matrix tablets of carvedilol. Nine formulations of matrix tablet were prepared using three hydrophilic polymers having $1 \%$ of three different dissolution enhancers. The matrix formers were sodium-carboxy methyl cellulose, Methocel K4M CR, Methocel K100M CR and the dissolution enhancers were PEG 6000, Poloxamer 188 and Kollidon-CLSF. Formulations F-10, F13 and F-16 contained PEG 6000 as dissolution enhancer, formulations F-11, F-14 and F-17 contained Poloxamer 188 and formulations F-12, F-15 and F-18 contained Kollidon-CLSF. Tablet granules were evaluated for bulk density $(0.293 \pm 0.012$ to $0.310 \pm 0.004 \mathrm{~g} / \mathrm{ml})$, tapped bulk density $(0.368 \pm 0.013$ to $0.380 \pm 0.012 \mathrm{~g} / \mathrm{ml})$ and compressibility index $(16.612 \pm 1.868$ to $22.834 \pm 5.426)$. The data indicated satisfactory flow properties of granules during compression. The tablets were subjected to thickness $(1.79 \pm 0.04 \mathrm{~mm})$, hardness $(11.46 \pm 1.06 \mathrm{~kg} / \mathrm{cm})$, and friability $(0.26 \pm 0.06 \%)$ measurements. The $i n$ vitro dissolution study was carried out for $12 \mathrm{hrs}$ using USP type II dissolution apparatus in 6.8 buffer as the dissolution medium where release mechanisms were subjected to zero order, first order, Korsmeyer-Peppas, Hixson-Crowell and Higuchi kinetic studies. The order of dissolution enhancing power was PEG $6000>$ Poloxamer $188>$ Kllidon-CLSF. The drug release from the tablets followed erosion mechanisms. Among all the formulation F-13, F-14 and F-17 exhibited USP complied in vitro dissolution profiles.
\end{abstract}

Key words: Matrix tablet, dissolution enhancer, carvedilol tablet.

\section{Introduction}

A perfect sustained release product is characterized by an initial release so that the plasma concentration of drug cross the minimum effective concentration quickly to give therapeutic activity, followed by gradual release of additional amounts of drug to maintain the plasma concentration in between minimum effective concentration and minimum toxic concentration. Matrix tablet is very popular in this regard. There are a number of factors associated with drug release from a matrix tablet, like- shape and size of matrix tablet, permeability of the pore, solubility of the drug and polymer, drug loading, hydrodynamic and conditions compression force (Veiga et al., 1988; Kim and Fassihi, 1997). Previous studies conducted by Williams et al. (2002) led to the conclusion that the type and level of excipients influence the rate and extension of drug release.

In the present investigation, studies were undertaken for the design and development of oral controlled drug delivery systems of an antihypertensive agent Carvedilol, through tablets, using the matrix diffusion technique. Carvedilol is well absorbed from the gastrointestinal tract but is subject to considerable first pass metabolism in the liver. Its absolute bioavailability is about $25 \%$ and plasma half-life is about 6 hours (McTavish, 1993). An attempt has been made to formulate carvedilol sustained release matrix tablet with the addition of $1 \%$ dissolution enhancer and to evaluate the effect 
dissolution enhancer on the release of carvedilol from the tablet matrix.

\section{Materials and Methods}

\section{Materials}

Carvedilol was received as gift sample from Incpeta Pharmaceuticals Ltd. Bangladesh. Merhocel K4M CR, Methocel K100M CR and Lactose were obtained from Colorcon, USA. Microcrystalline Cellulose (Avicel PH 102) was purchase from Hanau
Chemicals ltd. Japan. Magnesium stearate and talc were procured from Wilfrid Smith Ltd. UK. Poloxamer 188 and Kollidon-CLSF were obtained from BASF, Germany. PEG 6000 (Micronized grade) was donated by Beximco Pharmaceuticals Ltd, Bangladesh.

\section{Methods}

Formulation design: The tablets were formulated according to the table 1 as follows:

Table 1. Formulation of carvedilol by using dissolution enhancers.

\begin{tabular}{llllllllll}
\hline Ingredients & \multicolumn{9}{c}{ Formulation } \\
& 10 & 11 & 12 & 13 & 14 & 15 & 16 & 17 & 18 \\
\hline Carvedilol (mg) & 80 & 80 & 80 & 80 & 80 & 80 & 80 & 80 & 80 \\
Na-CMC (mg) & 33 & 33 & 33 & 45 & 45 & 45 & 0 & 0 & 0 \\
Methocel K4M (mg) & 45 & 45 & 45 & 0 & 0 & 0 & 45 & 45 & 45 \\
Methocel K100M (mg) & 0 & 0 & 0 & 33 & 33 & 33 & 33 & 33 & 33 \\
PEG 6000 (mg) & 3 & 0 & 0 & 3 & 0 & 0 & 3 & 0 & 0 \\
Poloxamer (mg) & 0 & 3 & 0 & 0 & 3 & 0 & 0 & 3 & 0 \\
Koliidon-CLSF (mg) & 0 & 0 & 3 & 0 & 0 & 3 & 0 & 0 & 3 \\
Lactose (mg) & 63 & 63 & 63 & 63 & 63 & 63 & 63 & 63 & 63 \\
Avicel PH 102 (mg) & 72.5 & 72.5 & 72.5 & 72.5 & 72.5 & 72.5 & 72.5 & 72.5 & 72.5 \\
Talc (mg) & 1.5 & 1.5 & 1.5 & 1.5 & 1.5 & 1.5 & 1.5 & 1.5 & 1.5 \\
Mg stearate (mg) & 2 & 2 & 2 & 2 & 2 & 2 & 2 & 2 & 2 \\
Total (mg) & 300 & 300 & 300 & 300 & 300 & 300 & 300 & 300 & 300 \\
\hline
\end{tabular}

Preparation of matrix tablets: In this study direct compression method has been applied for preparation of tablet matrix. Drug, polymer and other excipients were weighed separately for 10 tablets per formulation. Active ingredient, polymer, lactose, Avicel PH 102 and magnesium stearate were blended for 15 minutes and then talc was added and was blended for another 1 minute. The mixed mass was taken in the hopper and tablet compression was done to get the desired weight of the tablet (300mg). After compression, the tablets were weighed and tablet weight was found between $297.3 \mathrm{mg}-303.3 \mathrm{mg}$.
Characterization of carvedilol matrix tablets by evaluation of physical properties of formulation granules:

Bulk density: Both $L B D$ (Loose bulk density) and $T B D$ (Tapped bulk density) were determined by taking $2 \mathrm{gm}$ of granules in a $10 \mathrm{ml}$ measuring cylinder. After the initial volume was observed, the cylinder was placed into the tap density tester and the machine was set to a fixed rpm. Tapping was continued while waiting for no further change in volume of the granules. Using the following equations $L B D$ and $T B D$ were calculated (Shah et al., 1997): 
$L B D=$ Powder weight / Volume of the powder before tapping.

$T B D=$ Powder weight $/$ Volume of the powder after tapping

Compressibility index: The compressibility index of the granules was determined by Carr's compressibility index (Aulton, 1988):

Carr's compressibility index $(\%)=\frac{T B D-L B D}{L B D} \times 100$

Hausner ratio: It is very important parameter to be measured since it affects the mass of uniformity of the dose. It is measured using the following equation:

$$
\text { Hausner ratio }=\frac{T B D}{L B D}
$$

\section{Evaluation of physical properties of carvedilol matrix tablets}

Weight variation test: Twenty tablets from each formulation were subjected to weight variation test following official method.

Hardness: For each formulation, the hardness of 6 tablets was determined using the Dr. Schleuniger Pharmatron Tablet Tester.

Friability: Friability of 6 tablets of each proposed formulations were determined using the Erweka Friability Tester.

Thickness: Tablet thickness was determined using a thickness gauge. Five tablets from each batch were used, and average values were taken.

\section{In-vitro release studies of carvedilol matrix tablets}

In-vitro dissolution studies was performed in $900 \mathrm{ml}$ dissolution medium having $\mathrm{pH}$ 6.8. The temperature of the medium was maintained at $37 \pm 0.50{ }^{\circ} \mathrm{C}$. The USP type II apparatus was used and rpm (rotation per minute) was set to 100 (Sameer et al., 2009; Adamo et al., 2008; Chang et al., 2000; Hisakadzu and Yunxia, 2002). After 1hr, 2hr, 4hr, $8 \mathrm{hr}, 12 \mathrm{hr}$ definite volume $(5 \mathrm{ml})$ of aliquots were collected for analysis, which were then replaced with equal volume $\mathrm{pH} 6.8$ solution. The dissolution study was continued for 12 hours to get a simulated picture of the drug release and the percentage of drug release was plotted against time. This drug release profile was fitted into several mathematical models to get an idea of the release mechanism from the matrix tablets. The drug release at different time intervals was measured by a UV-visible spectrophotometer at $241 \mathrm{~nm}$ wavelength. The in vitro drug release kinetic data were tested with different mathematical models.

\section{Results and Discussion}

Flow properties of the granules: Generally, Carr's index values up to $16 \%$ and Hausner ratio values less than 1.25 are indicative of in good to excellent flow properties. According to the table 2, the Carr's index of most of the formulation were 16-23, while Hausner ration were below 1.25 which indicate good flow property. Thus, these were in suitable range for the preparation of tablets (Aulton, 2002).

Physical properties of carvedilol tablets: The physical properties of the prepared carvedilol tablets were determined. The diameter of the tablets were 13 $\mathrm{mm}$, thickness $1.79 \pm 0.04 \mathrm{~mm}$, hardness $11.46 \pm 1.06$, average weight $291.63 \pm 4.93 \mathrm{gm}$ to $301.57 \pm 4.51 \mathrm{gm}$ and friability were $0.26 \pm 0.06$. Thickness of all the tablets of all the formulations was found to be uniform. The average percentage of weight deviation of all tablet formulations was found to be within the limit. In this study, the percentage friability for all the formulations was below $1 \%$, indicating that the friability was within the prescribed limits. All the formulations showed acceptable compliance with the compendial specifications for weight variation, hardness and friability.

In-vitro dissolution and kinetic studies: All the tablet formulations were subjected to in-vitro drug release studies using $\mathrm{pH} 6.8$ buffer as dissolution medium, in order to assess drug release profiles including release kinetics and drug release mechanisms from tablets. It is evident from figures 1-5 that all the tablets gave a controlled release of carvedilol over a period of 12 hours. It can be inferred from the figures that most of the formulations will release the drug for about $24 \mathrm{hrs}$, because HPMC tablet formulations swelled upon contact with dissolution medium and a gel layer was 
formed on their surface. This gel retarded further ingress of fluid and subsequent drug release. After 12 hours the F10, F11 and F12 formulations showed $86.32 \%, 58.95 \%$ and $56.38 \%$ release respectively and F13, F14, F15 formulations showed $65.75 \%, 60.65 \%$ and $59.41 \%$ release respectively. F16, F17 and F18 formulations demonstrated $81.63 \%, 61.59 \%$ and
$39.03 \%$ release respectively. PEG 6000 (F10, F13 and F16) containing tablets showed more release than Poloxamer 188 (F11, F14, and F17) containing tablets and Kollidon-CLSF (F12, F15 and F18) containing tablets.

Table 2. Flow properties of the granules.

\begin{tabular}{lllll}
\hline Formulation & $\begin{array}{l}\text { Bulk } \\
\text { Density }(\mathrm{gm} / \mathrm{l})\end{array}$ & $\begin{array}{l}\text { Tapped } \\
\text { Density }(\mathrm{gm} / \mathrm{l})\end{array}$ & $\begin{array}{l}\text { Carr's } \\
\text { Index }(\%)\end{array}$ & Hausner Ratio \\
\hline 10 & $0.302 \pm 0.002$ & $0.373 \pm 0.010$ & $18.918 \pm 2.847$ & $1.234 \pm 0.044$ \\
11 & $0.295 \pm 0.014$ & $0.377 \pm 0.016$ & $21.642 \pm 6.779$ & $1.283 \pm 0.115$ \\
12 & $0.293 \pm 0.017$ & $0.380 \pm 0.005$ & $22.770 \pm 5.159$ & $1.299 \pm 0.090$ \\
13 & $0.304 \pm 0.006$ & $0.368 \pm 0.013$ & $17.295 \pm 4.425$ & $1.211 \pm 0.066$ \\
14 & $0.297 \pm 0.018$ & $0.376 \pm 0.016$ & $20.652 \pm 8.132$ & $1.269 \pm 0.132$ \\
15 & $0.298 \pm 0.010$ & $0.380 \pm 0.004$ & $21.468 \pm 2.198$ & $1.274 \pm 0.036$ \\
16 & $0.296 \pm 0.005$ & $0.379 \pm 0.003$ & $21.781 \pm 1.418$ & $1.279 \pm 0.023$ \\
17 & $0.296 \pm 0.016$ & $0.371 \pm 0.017$ & $20.097 \pm 7.791$ & $1.260 \pm 0.130$ \\
18 & $0.293 \pm 0.012$ & $0.380 \pm 0.012$ & $22.834 \pm 5.426$ & $1.300 \pm 0.095$ \\
\hline
\end{tabular}

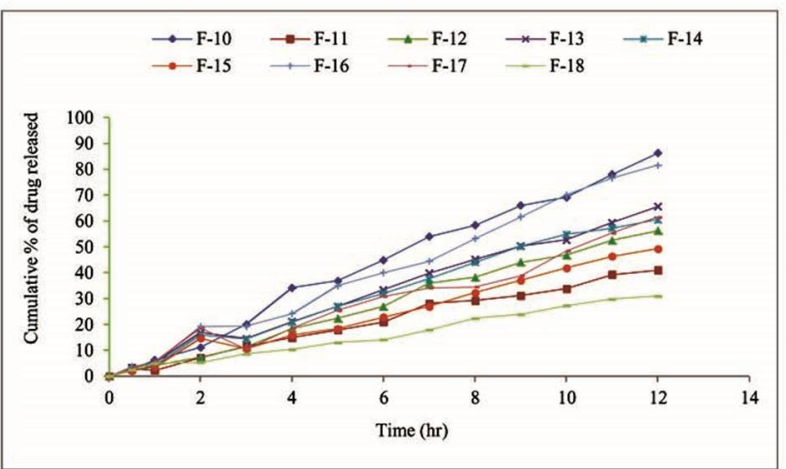

Figure 1. Zero order plot of release kinetics of formulations F-10 to F-18.

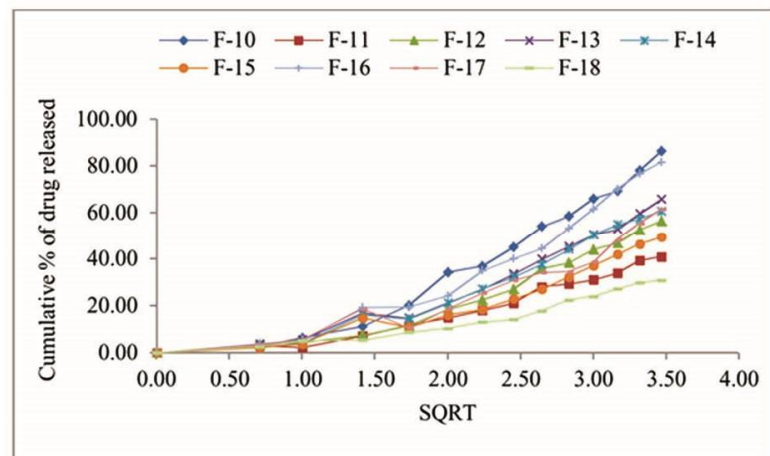

Figure 3. Higuchi plot of release kinetics of formulations F-10 to F-18.

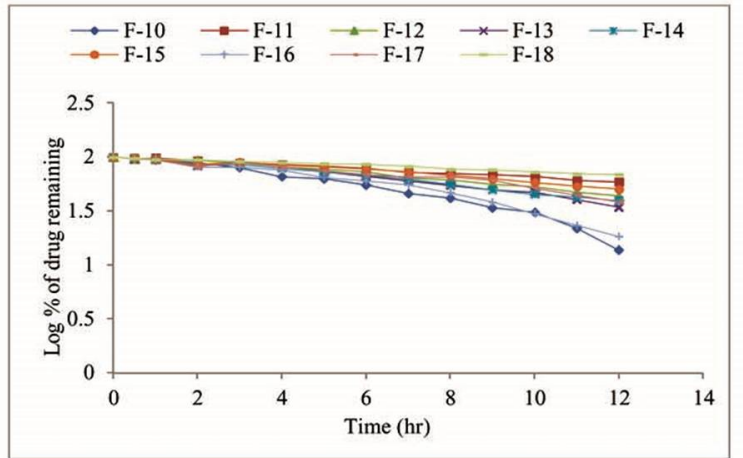

Figure 2. First order plot of release kinetics of formulations F-10 to F-18.

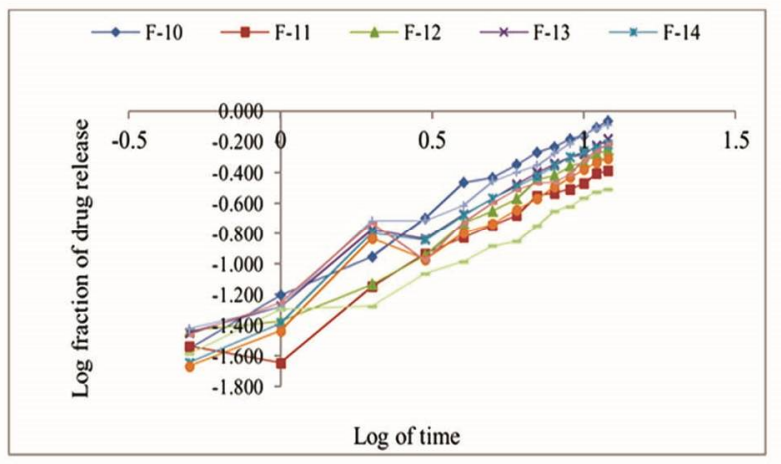

Figure 4. Korsmeyer-Peppas plot of release kinetics of formulations F-10 to F-18. 


\section{Release kinetics and mechanism}

The release rate constants of zero order, first order, Higuchi, Korsmeyer-Peppas and HixsonCrowell cube root law were calculated. In case of F-10 to F-18 Higuchi square root model showed correlation coefficients $0.898-0.938$ where F10 to F-16 followed Super Case II type of release and F-17 and F-18 followed Non-Fickian (Anomalous) type of release (Table 3).

Successive fractional dissolution time of the formulations of carvedilol matrix tablets was determined. $\mathrm{T}_{25 \%}, \mathrm{~T}_{50 \%}$ and $\mathrm{T}_{80 \%}$ were changed in line for the change of polymers ratio.

Table 3. Best fitted model for formulation F-10 to F-18.

\begin{tabular}{clcl}
\hline Formulation & Best fitted model & n value & Mechanism \\
\hline F10 & Zero order, Korsmeyer-Peppas, Hixson-Crowell & 1.085 & Super Case II transport \\
F11 & Korsmeyer-Peppas, Hixson-Crowell & 0.958 & Super Case II transport \\
F12 & Zero order, First order, Korsmeyer-Peppas & 0.959 & Super Case II transport \\
F13 & Zero order, Korsmeyer-Peppas, Hixson-Crowell & 0.929 & Super Case II transport \\
F14 & Zero order, First order, Korsmeyer-Peppas, Hixson-Crowell & 1.040 & Super Case II transport \\
F15 & Zero order, First order, Hixson-Crowell & 0.966 & Super Case II transport \\
F16 & Zero order, Korsmeyer-Peppas, Hixson-Crowell & 0.991 & Super Case II transport \\
F17 & Zero order & 0.876 & Anomalous/non - Fickian \\
& & & Transport \\
F18 & Zero order, Korsmeyer-Peppas, Hixson-Crowell & 0.784 & Anomalous/non - Fickian \\
& & & Transport \\
\hline
\end{tabular}

Table 4. Fractional dissolution time.

\begin{tabular}{lcccc}
\hline $\begin{array}{l}\text { Formu- } \\
\text { lation }\end{array}$ & $\begin{array}{c}\text { MDT } \\
(\mathrm{hr})\end{array}$ & $\begin{array}{c}\mathrm{T}_{25 \%} \\
(\mathrm{hr})\end{array}$ & $\begin{array}{c}\mathrm{T}_{50 \%} \\
(\mathrm{hr})\end{array}$ & $\begin{array}{c}\mathrm{T}_{80 \%} \\
(\mathrm{hr})\end{array}$ \\
\hline F-10 & 6.814 & 3.649 & 6.912 & 10.659 \\
F-11 & 14.640 & 7.039 & 14.513 & 23.705 \\
F-12 & 11.208 & 5.394 & 11.113 & 18.142 \\
F-13 & 9.358 & 4.369 & 9.214 & 15.281 \\
F-14 & 9.106 & 4.710 & 9.172 & 14.413 \\
F-15 & 12.568 & 6.090 & 12.481 & 20.302 \\
F-16 & 7.388 & 3.665 & 7.375 & 11.851 \\
F-17 & 11.205 & 4.930 & 10.877 & 18.600 \\
F-18 & 26.362 & 10.236 & 24.780 & 45.129 \\
\hline
\end{tabular}

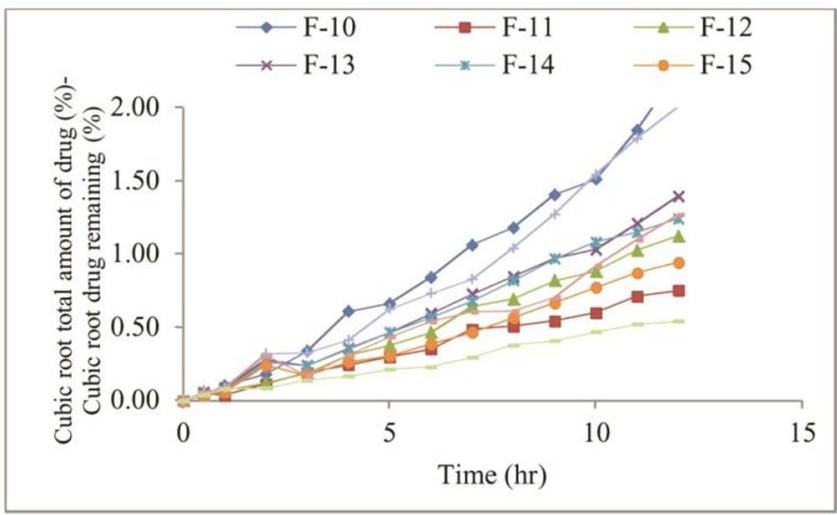

Figure 5. Hixson-Crowell plot of release kinetics of formulations F-10 to F-18.

Formulations which showed MDT values within 9 to 12 were considered as accepted for the sustained release for 24 hours. Most of the formulations showed MDT values between 10 and 12 hours. Among 9 formulations, 5 formulations gave MDT values within the accepted limit, 2 formulations (F-10 and F-16) gave MDT values below accepted limit and formulation F-11 and F-18 were found to be above the accepted limit (Table 4).

\section{Conclusion}

Carvedilol sustained release tablets were prepared with hydrophilic polymers- Methocel K4M CR, Methocel K100M CR, Methocel K 100M CR and sodium CMC by direct compression method. To enhance dissolution rate three different formulation sets (every set contain three different formulations) were developed using three different dissolution enhancer, namely PEG 6000, Poloxamer 188 and Kollidon -CLSF at $1 \%$ percentage. Among the 
dissolution enhancers, the rate of drug release was maximum in case of PEG 6000. The release rate of PEG 6000 (F-10, F-13, and F-16) containing tablets was more than Poloxamer 188 (F-11, F-14, and F-17) containing tablets and Kollidon-CLSF (F-12, F-15 and F-18) containing tablets. The formulations F-13, F-14 and F-17 successfully met the USP dissolution profile. Drug release kinetics indicated that the drug release was best explained by zero order, first order, Korsmeyer-Peppas and Hixson-Crowell, as these plots showed the highest linearity. KorsmeyerPappas plot indicated that the drug was released from the tablet matrices by erosion (> 0.89) and both diffusion and erosion mechanism $(\mathrm{n}>0.45$ but $\mathrm{n}<0.85$ ). Therefore, the optimization and development of carvedilol sustained release tablet were successful. It may conclude that by this study further investigation forgeting once daily dose can be developed.

\section{References}

Adamo, F., Valentina, B., Gian, C.C., Celestino, R., Carlos, A. 2008. Fast dispersible/slow releasing ibuprofen tablets. Eur. J. Pharm. and Biopharma. 69, 335-341.

Aulton, M.A. 2002. Pharmaceutics: The science of dosage form design, $2^{\text {nd }}$ Edition, pp. 133-134.
Chang, R.K., Guo, X., Burnside, B., Couch, R. 2000. Fastdissolving tablets. Pharmaceut. Technol. 24, 52-58.

Higuchi, T. 1963. Mechanism of sustained-action medication. Theoretical analysis of rate of release of solid drugs dispersed in solid matrices. J. Pharm. Sci. 52, 145-149.

Hisakadzu, S., Yunxia, B. 2002. Preparation, evaluation and optimization of rapidly disintegrating tablets. Powder Technol. 122, 188-198.

Kim, H., Fassihi, R. 1997. Application of binary polymer system in drug release rate modulation. Influence of formulation variables and hydrodynamic conditions on release kinetics. J. Pharm. Sci. 86, 323-328.

McTavish, D., Campoli-Richards, D. and Sorkin, E.M. 1993. Carvedilol: a review of its pharmacodynamic and pharmacokinetic properties and therapeutic efficacy. Drugs. 45, 232-258.

Sameer, G.L., Yi-Ying, Y. and Banga, A.K. 2009. Effects of disintegration-promoting agent, lubricants and moisture treatment on optimized fast disintegrating tablets, Intl. J. Pharmacy. 365, 4-11.

Veiga, F., Salsa, T. and Pina, M.E. 1988. Oral Controlled Release Dosage Forms II glassy polymers in hydrophilic matrices. Drug Dev. Ind. Pharm. 24, 1-9.

Williams III, R.O., Reynolds, T.D. and Cabelka, T.D. 2002. Investigation of excipient type and level on drug release from controlled release tablets containing HPMC. Pharm. Dev. Tech. 7, 181-193. 